\title{
Bunch lengthening by a betatron motion in quasi-isoch ronous storage rings
}

\author{
Yoshihiko Shoji \\ Laboratory of Advanced Science and Technology for Industry (LASTI), University of Hyogo, Ako-gun, Japan*
}

(Received 21 January 2004; published 28 September 2004)

\begin{abstract}
A simple analytical formula is described for bunch lengthening by a linear horizontal betatron motion in an electron storage ring. An example of calculation shows that this lengthening is larger than the intrinsic bunch shortening limit for most dispersive sections, which strongly limits the wavelength region of coherent radiation.
\end{abstract}

DOI: 10.1103/PhysRevSTAB.7.090703

PACS numbers: 29.20.Dh, 29.27.-a, 41.75.-i

\section{INTRODUCTION}

A quasi-isochronous electron synchrotron, which means a very small momentum compaction factor, can store a very short electron bunch. When its bunch length is a few mm or less, an extremely strong coherent synchrotron radiation in the $\mathrm{THz}$ frequency region is emitted even with a low stored beam current. Expanding on the observations of stable coherent radiation by Abo-Bakr et al. at BESSY-II [1], we examined new uses for this strong $\mathrm{THz}$ radiation. It is important to study limitations on the bunch shortening at a sufficiently low current. To date, two types of fundamental limitations in a linear system are theoretically predicted. One is the intrinsic bunch shortening limit from longitudinal radiation excitation [2]. The other is the effect of linear betatron motion. An electron in a storage ring passing through bending magnets at the outer side or inner side of a central orbit according to its betatron oscillation amplitude and phase has a deviation in the path length and produces a bunch lengthening. Deacon estimated this effect in 1981 [3], but he used a very rough approximation and did not consider that the lengthening depends on the location in the storage ring. I show that equations used to calculate synchrobeta resonance [4] give simple and accurate formulas for the bunch lengthening. I also show an example calculation using an isochronous lattice of an existing ring, NewSUBARU [5].

\section{THEORETICAL FORMULAS}

In this report my discussions are focused on the linear effect of the horizontal betatron motion. I assume that a vertical betatron motion and an energy displacement are negligible and higher order effects of betatron motion [6] are ignored. Coordinates $x$ and $s$ are the displacement from the reference electron in radial direction and the azimuthal coordinate, respectively. A displacement from the reference electron in the $s$ direction is referred to by $z$,

*Formerly Himeji Institute of Technology. the Twiss parameters by $\alpha(s), \beta(s)$, and $\gamma(s)$, and the betatron phase by $\psi(s)$.

The change in path length from the reference in one revolution $\delta L$ when higher order terms are ignored is given by

$$
\delta L=\int_{s_{S}}^{s_{S}+L_{0}}[x(s) / \rho(s)] d s
$$

Here $L_{0}$ is a circumference, $s_{S}$ is $s$ at the light source point, and $\rho(s)$ is the curvature of the radius of the reference orbit. When the radial displacement is produced by the betatron oscillation, $x(s)$ is written as

$$
x(s)=\sqrt{\varepsilon_{\mathrm{CSI}} \beta(s)} \sin \psi(s) .
$$

Here $\epsilon_{\mathrm{CSI}}$ is the Courant-Snyder invariant of a particle. If $s_{S} \leq s<s_{S}+L_{0}$ the betatron phase $\psi(s)$ after the $m$ revolutions around the ring is replaced by $\psi(s)+2 m \pi \nu$, where $\nu$ is the betatron tune. The total change of the path length after $n$ revolutions, $\delta L_{n}$, is given by

$$
\begin{aligned}
\delta L_{n}\left(s_{S}\right)= & \sqrt{\varepsilon_{\mathrm{CSI}}} \sum_{m=0}^{n-1} \int_{s_{S}}^{s_{S}+L_{0}}[\sqrt{\beta(s)} / \rho(s)] \sin [\psi(s) \\
& +2 m \pi \nu] d s .
\end{aligned}
$$

Here I will use the following equations,

$$
\begin{aligned}
& \sum_{m=0}^{n-1} \sin \left[(2 m+1) \pi \nu+\psi\left(s_{S}\right)\right] \\
& =(\sin n \pi \nu / \sin \pi \nu) \sin \left[n \pi \nu+\psi\left(s_{S}\right)\right], \\
& \sum_{m=0}^{n-1} \cos \left[(2 m+1) \pi \nu+\psi\left(s_{S}\right)\right] \\
& \quad=(\sin n \pi \nu / \sin \pi \nu) \cos \left[n \pi \nu+\psi\left(s_{S}\right)\right],
\end{aligned}
$$

dispersion function $\eta(s)$, and dispersion angle $\eta^{\prime}(s)$ given by

2004 The American Physical Society

090703-1 


$$
\begin{aligned}
\eta\left(s_{S}\right) & =\frac{\sqrt{\beta\left(s_{S}\right)}}{2 \sin \pi \nu} \int_{s_{S}}^{s_{S}+L_{0}} \frac{\sqrt{\beta(s)}}{\rho(s)} \cos \left[\psi(s)-\psi\left(s_{S}\right)-\pi \nu\right] d s, \\
\alpha\left(s_{S}\right) \eta\left(s_{S}\right)+\beta\left(s_{S}\right) \eta^{\prime}\left(s_{S}\right) & =\frac{\sqrt{\beta\left(s_{S}\right)}}{2 \sin \pi \nu} \int_{s_{S}}^{s_{S}+L_{0}} \frac{\sqrt{\beta(s)}}{\rho(s)} \sin \left[\psi(s)-\psi\left(s_{S}\right)-\pi \nu\right] d s .
\end{aligned}
$$

With these equations Eq. (3) is simplified to [4]

$$
\begin{aligned}
\delta L_{n}\left(s_{S}\right)= & 2 \sin (n \pi \nu) \sqrt{\varepsilon_{\mathrm{CSI}}}\left\{\left[\eta\left(s_{S}\right) / \sqrt{\beta\left(s_{S}\right)}\right] \sin [n \pi \nu\right. \\
& \left.+\psi\left(s_{S}\right)\right]+\left[\eta\left(s_{S}\right) \alpha\left(s_{S}\right) / \sqrt{\beta\left(s_{S}\right)}+\eta^{\prime}\left(s_{S}\right)\right. \\
& \left.\left.\times \sqrt{\beta\left(s_{S}\right)}\right] \cos \left[n \pi \nu+\psi\left(s_{S}\right)\right]\right\} .
\end{aligned}
$$

If functions $H(s)$ and $\psi_{H}(s)$ are defined by

$$
\begin{aligned}
\sqrt{H} \sin \psi_{H} & =\eta(\alpha / \sqrt{\beta})+\eta^{\prime} \sqrt{\beta}, \\
\sqrt{H} \cos \psi_{H} & =\eta / \sqrt{\beta},
\end{aligned}
$$

Eq. (6) can be rewritten as

$$
\begin{aligned}
\delta L_{n}\left(s_{S}\right)= & \sqrt{\varepsilon_{\mathrm{CSI}} H\left(s_{S}\right)}\left\{-\cos \left[2 n \pi \nu+\psi\left(s_{S}\right)+\psi_{H}\left(s_{S}\right)\right]\right. \\
& \left.+\cos \left[\psi\left(s_{S}\right)+\psi_{H}\left(s_{S}\right)\right]\right\} .
\end{aligned}
$$

The function $H$ is given by

$$
H=\gamma \eta^{2}+2 \alpha \eta \eta^{\prime}+\beta \eta^{\prime 2}
$$

The longitudinal displacement from the reference $z$ after $n$ revolutions is given by

$$
z=-\delta L_{n}-z_{S}
$$

Here $z_{S}$ is an initial displacement, which depends on the initial phase $\psi\left(s_{S}\right)$. If the reference is defined as an average over $\psi\left(s_{S}\right), z_{S}$ is determined and the longitudinal displacement $z\left(s_{S}\right)$ is given by

$$
z\left(s_{S}\right)=\sqrt{\varepsilon_{\mathrm{CSI}} H\left(s_{S}\right)} \cos \left[2 n \pi \nu+\psi\left(s_{S}\right)+\psi_{H}\left(s_{S}\right)\right],
$$

where the radial displacement $x\left(s_{S}\right)$ is given by

$$
x\left(s_{S}\right)=\sqrt{\varepsilon_{\mathrm{CSI}} \beta\left(s_{S}\right)} \sin \left[2 n \pi \nu+\psi\left(s_{S}\right)\right] .
$$

Notice that $H$ and $\psi_{H}$ are unique functions of $s_{S}$ but $\psi$ is not. Equation (9) shows that the amplitude of the oscillation of $z$ is $\sqrt{\varepsilon_{\mathrm{CSI}}} \bar{H}$. The bunch lengthening is zero at a dispersion free location. The $\psi_{H}-\pi / 2$ is the phase difference between the radial and the longitudinal oscillations. The particle moves on an ellipse in the $z-x$ plane. When $\psi_{H}$ is $\pm \pi / 2$ the ellipse shrinks to a line.

The density distribution function in the $z$ axis of a bunch of particles with the same $\epsilon_{\mathrm{CSI}}$ and uniform distribution in $\psi_{S}$ is given by

$$
D\left(z, \varepsilon_{C S I}\right) d z= \begin{cases}\frac{1}{\pi} \frac{d z}{\left(\varepsilon_{\mathrm{CSI}} H-z^{2}\right)} & \text { for } z^{2}<\varepsilon_{\mathrm{CSI}} H \\ 0 & \text { for } z^{2} \geq \varepsilon_{\mathrm{CSI}} H .\end{cases}
$$

In a realistic model, the particles have $\epsilon_{\mathrm{CSI}}$ variations and the density distribution function in the $\epsilon_{\mathrm{CSI}}$ axis is

$$
F\left(\varepsilon_{\mathrm{CSI}}\right) d \varepsilon_{\mathrm{CSI}}=\frac{1}{2 \varepsilon} e^{-\varepsilon_{\mathrm{CII}} / 2 \varepsilon} d \varepsilon_{\mathrm{CSI}}
$$

Here a Gaussian distribution is assumed in a betatron oscillation phase space with $\mathrm{rms}$ emittance of $\varepsilon$. The realistic distribution is given by a convolution of Eq. (13) with Eq. (14),

$$
\begin{aligned}
G(z) d z & =\left[\int_{0}^{\sqrt{\varepsilon H}} F\left(\varepsilon_{\mathrm{CSI}}\right) D\left(z, \varepsilon_{\mathrm{CSI}}\right) d \varepsilon_{\mathrm{CSI}}\right] d z \\
& =\frac{1}{\sqrt{2 \pi} \varepsilon H} e^{-z^{2} / 2 \varepsilon H} d z .
\end{aligned}
$$

It is a Gaussian distribution with standard deviation of $\sqrt{\varepsilon} \bar{H}$.

\section{CALCULATION AT NEWSUBARU}

NewSUBARU is a $1.5 \mathrm{GeV}$ racetrack-type synchrotron radiation ring at the SPring-8 site. Its bending cell is a modified double bend achromat with one $-8^{\circ}$ invert bend between two $34^{\circ}$ normal bends. This cell facilitates control of the linear momentum compaction factor $\left(\alpha_{p}\right)$ while maintaining the achromatic cell with only a small change in the natural emittance. Table I lists the parameters of the ring used in the calculation when the ring was operated in quasi-isochronous operation mode [7].

Figure 1 shows the horizontal beta function $\beta$ and the dispersion function $\eta$ in $1 / 4$ of the ring. Figure 2 shows the $H$ and the $\psi_{H}$ in $1 / 4$ of the ring. The maximum rms bunch lengthening by the natural emittance $\left(\varepsilon_{X N}\right)$ is $0.10 \mathrm{~mm}$ at the inverse bends. The bunch lengthening was $0.06 \mathrm{~mm}$ at the light source point of the beam line used to measure the bunch length.

TABLE I. Main parameters of NewSUBARU.

\begin{tabular}{lc}
\hline \hline Stored electron energy $E(\mathrm{GeV})$ & 1.0 \\
Circumference $L_{0}(\mathrm{~m})$ & 118.73 \\
rf frequency $f_{\mathrm{rf}}(\mathrm{MHz})$ & 499.956 \\
Natural emittance $\varepsilon_{X N}(\mathrm{~nm})$ & 30.1 \\
Horizontal betatron tune $\nu$ & 6.30 \\
Linear momentum compaction factor $\alpha_{p}$ & 0 \\
Natural energy spread $\sigma_{\mathrm{EN}}(\%)$ & 0.047 \\
Intrinsic bunch shortening limit $\sigma_{\mathrm{TI}}(\mathrm{ps})$ & 0.08 \\
\hline \hline
\end{tabular}




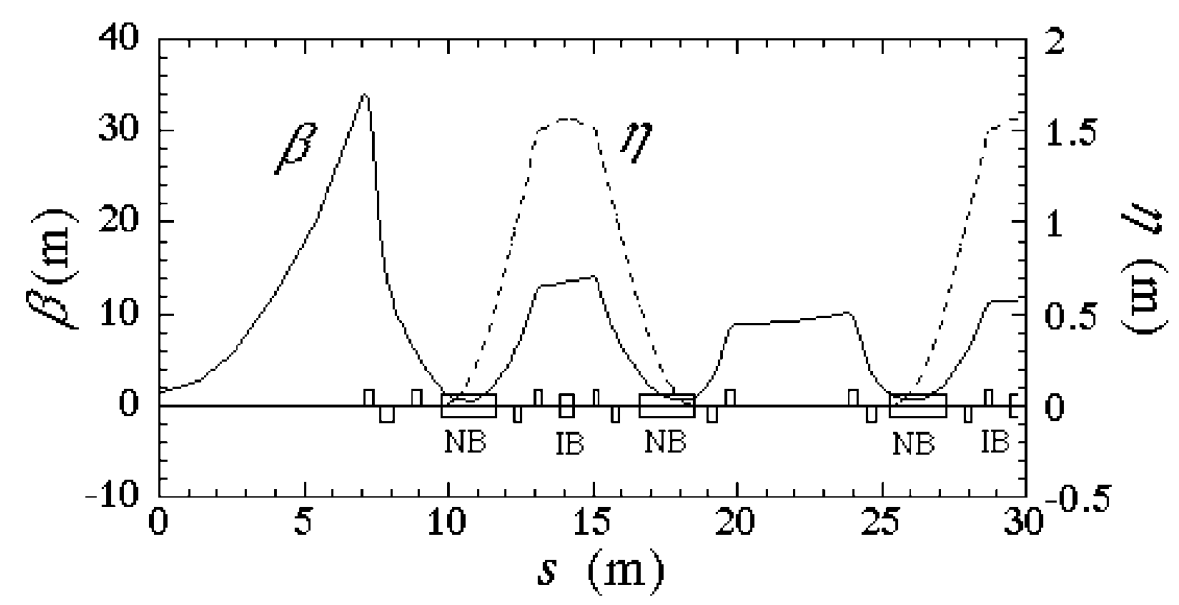

FIG. 1. Beta function $(\beta$, solid line) and dispersion function $(\eta$, broken line) in $1 / 4$ of NewSUBARU. The boxes indicate locations and lengths of the bending magnets (indicated by NB and IB) and quadrupole magnets.

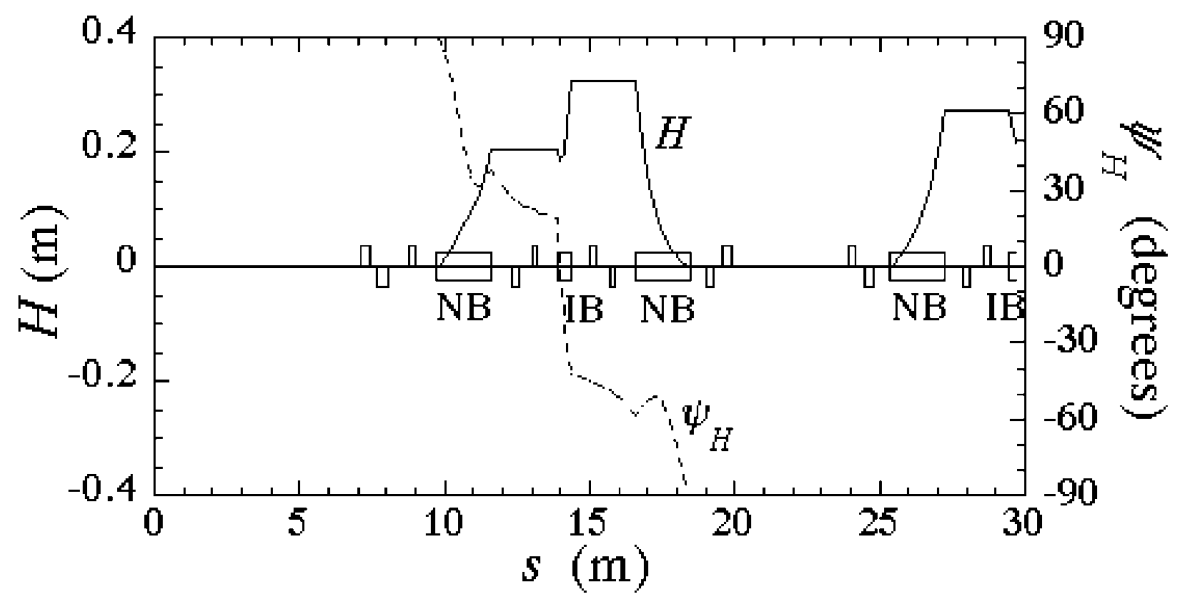

FIG. 2. The amplitude function $\left(H\right.$, solid line) and the phase difference between the longitudinal and transversal oscillation $\left(\psi_{H}\right.$, broken line) along $1 / 4$ of NewSUBARU.

\section{DISCUSSION}

This bunch lengthening effect should be considered in extreme cases beyond those realized at BESSY-II $\left(\sigma_{L}=\right.$ $0.45 \mathrm{~mm})[8]$ and at NewSUBARU $\left(\sigma_{L}=0.48 \mathrm{~mm}\right)$. For coherent radiation Eq. (15b) gives a strong limitation to the wavelength of radiation. The time structure of a bunch, produced by the rf potential well distortion or any method, is smeared in bending magnets.

I will compare the calculated $\sqrt{\varepsilon_{X N}} \bar{H}$ with the intrinsic bunch shortening limit coming from the longitudinal radiation excitation. The idea is that a stochastic fluctuation where the photoemission takes place produces a fluctuation of $\mathrm{rf}$ phase and enlarges the equilibrium bunch length and energy spread as the energy spread excitation does. Because of this radiation excitation the bunch length cannot be larger than

$$
\sigma_{\mathrm{TI}}=T_{0} \sigma_{\mathrm{EN}} \sqrt{I_{\alpha}}
$$

at any locations of the ring. This is the intrinsic limit of a storage ring determined only by $T_{0}$ (revolution period), $\sigma_{\mathrm{EN}}$ (natural energy spread), and $I_{\alpha}$ [a variance of partial momentum compaction factor: $\breve{\alpha}(s)]$. The $I_{\alpha}$ and $\breve{\alpha}(s)$ are given by the following equations:

$$
\begin{gathered}
\breve{\alpha}\left(s_{S}\right)=\frac{1}{L_{0}} \int_{s_{S}}^{L_{0}} \frac{\eta(s)}{\rho(s)} d s, \\
I_{\alpha}=\left\langle\left[\breve{\alpha}\left(s_{S}\right)-\langle\breve{\alpha}\rangle\right]^{2}\right\rangle .
\end{gathered}
$$


In NewSUBARU $\sigma_{\mathrm{TI}}$ is calculated to be $0.02 \mathrm{~mm}$, which is smaller than $\sqrt{\varepsilon_{X N} H}$ in most locations of the bending magnets.

\section{ACKNOWLEDGMENTS}

The author thanks Dr. S. Daté, Mr. T. Nakamura, and Dr. H. Tanaka of SPring-8 for their helpful discussions on this subject and proofreading this article.

[1] M. Abo-Bakr, J. Feikes, K. Holldack, P. Kuske, W. B. Peatman, U. Schade, G. Wüstefeld, and H.W. Hübers, Phys. Rev. Lett. 90, 094801 (2003).

[2] Y. Shoji, H. Tanaka, M. Takao, and K. Soutome, Phys. Rev. E 54, R4556 (1996).

[3] David A. G. Deacon, Phys. Rep. 76, 349 (1981); see, in particular, p. 385.
[4] A. Piwinski, and A. Wrulich, DESY Report No. 76/71, 1976; A. Piwinski, Handbook of Accelerator Physics and Engineering, edited by A. Wu Chao and M. Tigner (World Scientific, Singapore, 1999), Chap. 2, p. 72.

[5] A. Ando, A. Amano, S. Hashimoto, H. Kinoshita, S. Miyamoto, T. Mochizuki, M. Niibe, Y. Shoji, M. Terasawa, T. Watanabe, and N. Kumagai, J. Synchrotron Radiat. 5, 342 (1998).

[6] Helmut Wiedemann, Particle Accelerator Physics II (Springer-Verlag, Berlin, 1995), Chap. 6, p. 208.

[7] Y. Shoji, A. Ando, and S. Hashimoto, in Eighth International Conference on Synchrotron Radiation Instrumentation, edited by $\mathrm{T}$. Warwick et al., AIP Conf. Proc. No. 705 (AIP, New York, 2004), p. 57.

[8] M. Abo-Bakr, W. Anders, P. Kuske, and G. Wüsterfeld, in Proceedings of the Particle Accelerator Conference, Portland, OR, 2003 (IEEE, Piscataway, NJ, 2003), p. 3020. 